\title{
Rechtsgeschichte
}

http://www.rg-rechtsgeschichte.de/rg7

$\operatorname{Rg} / 2005 \quad 191-193$

Zitiervorschlag: Rechtsgeschichte Rg 7 (2005)

http://dx.doi.org/10.12946/rg07/191-193

\section{Stefan-Ludwig Hoffmann}

\section{Opfer des Faschismus und des Antifaschismus}




\section{Opfer des Faschismus und des Antifaschismus*}

Wer nach der Befreiung der Konzentrationslager im Winter und Frühjahr I945 als ein Opfer des Faschismus galt, das verstand sich nicht von selbst und bleibt in gewisser Weise bis heute Gegenstand vergangenheitspolitischer Kontroversen. Nach Kriegsende waren es zunächst die politischen Häftlinge, die für sich den Status ১Opfer des Faschismus beanspruchten und eine Heroisierung des antifaschistischen Widerstandes betrieben. Die europäischen Juden aber, die im engeren Sinne keine politischen Gegner des Nationalsozialismus waren, sondern snur aufgrund rassistischer Kriterien verfolgt und vernichtet wurden, blieben anfangs aus der symbolpolitischen Gemeinschaft der >Opfer des Faschismus ausgeschlossen - eine Sichtweise, die sich wiederum bis heute nahezu umgekehrt hat. Doch der Vernichtungswillen des nationalsozialistischen Deutschlands galt auch Gruppen der Gesellschaft, die sich zur Heroisierung wenig eigneten: >Asozialen`, >Kriminellen`, Homosexuellen oder geistig Behinderten. Dem Umgang mit diesen vermeintlich unwürdigen Opfern im Berlin der unmittelbaren Nachkriegszeit hat Susanne zur Nieden eine konzise Fallstudie gewidmet.

Der erste Teil des Buches rekonstruiert, zum Teil im Rückgriff auf die Forschungsliteratur, die Berliner Anerkennungs- und Entschädigungspolitik der Opfer der nationalsozialistischen Gewaltherrschaft der Jahre I945 bis I948/49. Während die Millionen `Displaced Persons`, die sich nach Kriegsende in Europa befanden, der Fürsorge der Vereinten Nationen und der westlichen Alliierten unterstanden, waren die deutschen NS-Verfolgten auf die deutschen Behörden angewiesen. In dieser Situation gingen sie den
Weg der Selbstorganisation, um ihre Interessen wahrzunehmen. Bereits im Mai 1945 entstand der Berliner Hauptausschuss >Opfer des Faschismus`, mit dem KZ-Überlebenden und Kommunisten Ottomar Geschke, der zugleich Stadtrat für Soziales war, an der Spitze. Während andere spontan gegründete Antifa-Ausschüsse bald von den neuen Machthabern aufgelöst wurden, konnte der Hauptausschuss sich als politisches Gremium wie auch als eine Art amtliche Wohlfahrtsstelle aller in Berlin lebenden NS-Verfolgten behaupten. Der Hauptausschuss geriet bald in den Strudel der politischen Auseinandersetzung zwischen Ost und West. In der DDR behielt er - und damit die Überlebenden der Lager selbst - die Definitionsmacht darüber, wer ein Opfer des Faschismus war und wer nicht. In der Bundesrepublik entschieden über die Entschädigung letztlich Verwaltungsbehörden, in der viele alte Eliten des `Dritten Reiches` weiter wirkten.

Dass die Anerkennungs- und Entschädigungspraxis damit selbst Gegenstand des Kalten Krieges wurde, kann nicht überraschen und wurde, etwa in den Arbeiten von Constantin Goschler, bereits ausführlich beschrieben. So fielen Kommunisten oftmals im Westen bald aus der Entschädigungspraxis heraus, weil sie an der Errichtung einer neuen totalitären Gewaltherrschaft arbeiten würden, wie umgekehrt die Gegner des Antifaschismus der DDR, vor allem die Nichtkommunisten unter den KZ-Überlebenden, selbst zu Faschisten erklärt wurden. Noch irritierender ist der Befund, dass in beiden deutschen Nachkriegsgesellschaften die gleichen Gruppen von NS-Verfolgten keine Chance auf Wiedergutmachung hatten, jene Gruppen, die nicht aus politischen oder rassistischen Gründen

\footnotetext{
* Susanne zur Nieden, Unwürdige Opfer. Die Aberkennung von NS-Verfolgten in Berlin 1945 bis I949, Berlin: Metropol Verlag 2003, 208 S., ISBN 3-9364I I-20-4
} 
verfolgt wurden, jedenfalls nach den Begriffen von Politik und 'Rasse`, die in der Nachkriegszeit vorherrschend waren.

Das zeigt eindrucksvoll der zweite, empirisch dichtere Teil der Studie, der eine als 'Säuberung deklarierte Überprüfung durch den Berliner Hauptausschuss zum Gegenstand hat, bei der in den Jahren zwischen I946 und 1949 über 700 politische Verfolgte (von insgesamt 9.000) ihren Status als anerkannte Opfer des Faschismus verloren. Die überlieferten Akten der individuellen Fallprüfung durch den Hauptausschuss legen die Kriterien frei, nach denen die ehemaligen politischen Häftlinge der Konzentrationslager darüber entschieden, wer in das Passepartout des antifaschistischen Heroismus passte. Die Kriterien geben mithin indirekt Auskunft über die Wert- und Ordnungsvorstellungen eines Teils der Überlebenden, der sich als politische Widerstandskämpfer sah.

Dieses politische Selbstverständnis besaß eine Vorgeschichte in der Ordnung der Lager. So wurden gleichgeschlechtliche Beziehungen, gefördert zumal durch die jahrelange Internierung, von den politischen Häftlingen strikt abgelehnt und schon in den Lagern sanktioniert. Da man um das öffentliche Ansehen der Opfer des Faschismus ängstlich besorgt war, besaßen in der Nachkriegszeit die Vorurteilsstrukturen der deutschen Bevölkerung für die Entscheidungen des Hauptausschusses ebenfalls eine nicht zu unterschätzende Bedeutung. Das zeigt die Studie exemplarisch an einzelnen Fällen. So erfuhr der Prüfer des Hauptausschusses von der Berliner Oberstaatsanwaltschaft im November 1946 von einer Anzeige gegen zwei Schwestern, beide Auschwitz-Überlebende. Die Vermieterin der Wohnung hatte die beiden Schwestern angezeigt, nachdem eine von ihnen ein Verhältnis mit der volljährigen Tochter der Vermieterin begonnen hatte. Zwar wurde das Verfahren von der Staatsanwaltschaft eingestellt, da der Paragraph I75 des Strafgesetzbuches ausschließlich gleichgeschlechtliche Beziehungen zwischen Männern unter Strafe stellte, der Hauptausschuss wurde dennoch aktiv. Im Zuge der Überprüfung stellte sich heraus, dass die beiden Schwestern im Sommer 1945 bei der ersten Anerkennung als Opfer des Faschismus fälschlicherweise angegeben hatten, sie seien aufgrund politischen Widerstandes verhaftet worden. Zu diesem Zeitpunkt besaß der Verweis auf ihre jüdische Herkunft keine Chance auf Anerkennung als Opfer. Noch bevor der Ausschuss zu einer endgültigen Entscheidung kam, entzogen sich die beiden Schwestern durch Emigration in die Vereinigten Staaten dem Richterspruch der selbsternannten Sittenwächter.

Weniger Skrupel zeigte der Hauptausschuss bei anderen Formen sunwürdigen Verhaltens. Wer aufgrund von Homosexualität, geistiger Behinderung oder sasozialem < Lebenswandel in die KZs kam, konnte auf eine Anerkennung als Opfer des Faschismus nur hoffen, wenn er die Verfolgung als politische ausgab. Und wer nach I945 teilhatte an der weit verbreiteten Beschaffungskriminalität der Nachkriegszeit, an der praktisch alle Schichten der Gesellschaft partizipierten, musste ebenfalls mit Aberkennung seines Opferstatus rechnen. Die genaue Rekonstruktion einzelner Fallbeispiele zeigt nicht nur die Bruchlinien innerhalb der Gemeinschaft des Lagers, die sich gleichsam in die Nachkriegszeit verlängerten. Sie bietet auch einen Einblick in das soziale Flechtwerk der Umbruchsgesellschaft zwischen Befreiung und Kaltem Krieg, in der die vom Nationalsozialismus Deklassierten nun versuchen sollten, die Stereotypen der Verfolgung durch eine Lebensführung zu widerlegen, die an den sozialen und moralischen Realitäten, zumal 
der Zusammenbruchsgesellschaft, völlig vorbeiging. Folglich sollte auch das gegenwärtige Anliegen einer nachholenden Erinnerung an vergessene Opfergruppen des Nationalsozialismus die Vielschichtigkeit der Verfolgtenbiographien nicht aus dem Blick verlieren. Zur Nieden entgeht der Gefahr neuer Stilisierungen dadurch, dass sie auch jene Fälle behandelt, in denen ehemalige Häftlinge der Konzentrationslager sich wechselseitig Verbrechen gegen die Menschlichkeit vorwarfen, mithin die Grenze zwischen Opfern und Tätern nicht scharf gezogen war.

Die Perspektive für künftige Studien, die zur Nieden implizit freilegt, zielt auf eine diachrone Längsschnittanalyse eines Diskurses über sge- borene Verbrecher`, ssexuelle Devianz` und ‘unwertes Leben`, der lange vor dem Nationalsozialismus, im bürgerlichen I9. Jahrhundert, einsetzt und weit in die Nachkriegszeit der beiden deutschen Gesellschaften hineinreicht. Diesem Diskurs über abweichendes Verhalten konnten und wollten sich die politischen Gegner des Faschismus nicht entziehen. Nicht nur in dieser Hinsicht war das Wertesystem des neu geschaffenen Arbeiter- und Bauernstaates im Osten Deutschlands, an dessen Spitze bis zuletzt viele politische Widerstandskämpfer standen, ’bürgerlicher` als gemeinhin angenommen.

Stefan-Ludwig Hoffmann

\section{Eine Entscheidung, die vom Himmel fiel*}

Der Bundesgerichtshof hat im Jahre 1954 im sogenannten "Schacht-Leserbriefentscheid " ${ }^{I}$ das allgemeine Persönlichkeitsrecht als »sonstiges Recht « im Sinne des $\mathbb{8} 823$ Abs. I BGB anerkannt. Es handelte sich um eine - zumindest für reine Gesetzespositivisten - skandalöse praeter oder extra legem-Entscheidung. Eine Münchner Dissertation, entstanden bei Hermann Nehlsen, widmet sich, rund 50 Jahre nach diesem Markstein richterlicher Rechtsfortbildung, der Vorgeschichte. Sie untersucht, wie es soweit hat kommen können, und legt dar, dass diesem Urteil eine jahrzehntelange, wechselvolle und spannende Diskussion in der juristischen Lehre voranging.

Es liegt nahe, im BGH-Urteil vom 25. Mai I954 den Endpunkt einer rechtshistorischen Entwicklung in einem - durchaus wichtigen - Bereich zu sehen. Hatte das Persönlichkeitsrecht die deutschen Juristen doch nicht nur seit Inkrafttreten des BGB, sondern - wie man eindrücklich erfährt - weit über ıoo Jahre zuvor beschäftigt. Die Autorin scheut nun - zu Recht - davor zurück, die Nachweise (spät-)naturrechtlicher Diskussionsbeiträge des ausgehenden I8. Jahrhunderts oder die eingehend von ihr geschilderten juristischen Positionen des I9. Jahrhunderts dafür zu bemühen, die BGH-Entscheidung als unausweichlich zu begründen. Schließlich steht am Ende dieser Phase das BGB von I900, welches gerade kein allgemeines Persönlichkeitsrecht anerkannte.

Das hinderte allerdings die deutschen Juristen (und zum Teil dieselben wie zuvor!) keineswegs daran, die Frage weiter intensiv zu erörtern und sich für und wider eine Anerkennung eines solchen Rechts auszusprechen. Der »Wille des Gesetzgebers « hat der Diskussion in der Lehre

\footnotetext{
* Katrin Kastl, Das allgemeine Persönlichkeitsrecht. Der Prozess seiner Anerkennung als »sonstiges Recht « im Sinne von $\$ 823$ Abs. I BGB (Abhandlungen zur rechtswissenschaftlichen Grundlagenforschung, Bd. 92), Ebelsbach: Aktiv Druck 2004, XVI, 292 S., ISBN 3-932653-I7-3

I Zur Einnerung: Der frühere

Reichsbankpräsident und Wirtschaftsminister des Dritten Rei-
}

ches, Dr. Hjalmar Schacht, wurde im Nürnberger Prozess freigesprochen und gründete 1952 eine Privatbank. Dies war Anlass für die »Welt am Sonntag « (WamS), seine politische Vergangenheit zu beleuchten, worauf der Anwalt Schachts, Müller, einen Berichtigungsanspruch für seinen Mandanten geltend machte. Die »WamS « druckte den Text so ab, dass er als bloßer Leserbrief des
Anwalts erschien, der presserechtliche Hintergrund seiner Intervention und das Mandatsverhältnis zu Schacht blieben dem Zeitungsleser damit verborgen. Der Anwalt setzte nun seinerseits aus eigenem Recht durch, dass die "WamS « ihre Behauptung, Anwalt Müller habe ihr einen Leserbrief in Sachen Dr. Schacht gesandt, zurücknehmen musste - dogmatische Grundlage war das mit dieser 